\title{
Fuzuloparib: First Approval
}

\author{
Arnold Lee ${ }^{1}$ \\ Published online: 12 June 2021 \\ (C) Springer Nature 2021, corrected publication 2021
}

\begin{abstract}
Fuzuloparib (AiRuiYi ${ }^{\circledR}$, 艾瑞臨; formerly fluzoparib) is a small molecule, orally active PARP inhibitor being developed by Jiangsu Hengrui Pharmaceuticals Co., Ltd. (formerly Jiangsu Hengrui Medicine Co., Ltd.) for the treatment of solid cancers. Fuzuloparib has been approved in China for the treatment of ovarian cancer (including fallopian tube cancer or primary peritoneal cancer), and phase II and III trials are investigating fuzuloparib for the treatment of other solid cancers, including cancers of the pancreas, breast, prostate and lungs. This article summarizes the milestones in the development of fuzuloparib leading to this first approval for the treatment of platinum-sensitive recurrent ovarian cancer, fallopian tube cancer or primary peritoneal cancer in patients with germline $B R C A$ mutation who have undergone second-line or above chemotherapy.
\end{abstract}

\section{Digital Features for this AdisInsight Report can be found at https:// doi.org/10.6084/m9.figshare.14691351.}

\section{Fuzuloparib (AiRuiYi ${ }^{\oplus}$, 艾瑞颐): Key points}

A small molecule PARP inhibitor is being developed by Jiangsu Hengrui Pharmaceuticals Co., Ltd. (formerly Jiangsu Hengrui Medicine Co., Ltd.) for the treatment of ovarian cancer and other solid cancers

Received its first approval on 11 Dec 2020 in China

Approved for use in platinum-sensitive recurrent ovarian cancer, fallopian tube cancer or primary peritoneal cancer in patients with germline $B R C A$ mutation who have undergone second-line or above chemotherapy
This profile has been extracted and modified from the AdisInsight database. AdisInsight tracks drug development worldwide through the entire development process, from discovery, through preclinical and clinical studies to market launch and beyond.

Arnold Lee

dru@adis.com

1 Springer Nature, Private Bag 65901, Mairangi Bay, Auckland 0754, New Zealand

\section{Introduction}

Fuzuloparib (AiRuiYi ${ }^{\circledR}$, 艾瑞臨; formerly fluzoparib) is a PARP inhibitor being developed by Jiangsu Hengrui Pharmaceuticals Co., Ltd. (formerly Jiangsu Hengrui Medicine Co., Ltd.) for the treatment of recurrent ovarian cancer (including fallopian tube cancer or primary peritoneal cancer) and other solid cancers. Fuzuloparib is the first original PARP inhibitor to be developed in China and it expands the number of PARP inhibitors available for the treatment of cancer [1]. Fuzuloparib received its first approval on 11 Dec 2020 in China for the treatment of platinum-sensitive recurrent ovarian cancer, fallopian tube cancer or primary peritoneal cancer (hereafter referred to as ovarian cancer) in patients with germline $B R C A$ mutation $(g B R C A m)$ who have undergone second-line or above chemotherapy [1-3]. Fuzuloparib is not yet indicated for maintenance therapy of ovarian cancer [3], however, development in this indication is ongoing.

The recommended dosage of fuzuloparib is $150 \mathrm{mg}$ taken orally twice daily. Detection of a harmful or suspected harmful gBRCA1/2 mutation using a method approved by the National Medical Products Administration is required in China prior to starting treatment with fuzuloparib. Reducing the dose to $50 \mathrm{mg}$ is recommended with the concurrent administration of a moderate CYP3A4 inhibitor; concurrent administration with a strong CYP3A4 inhibitor or inducer is not recommended. Dose reduction or interruption may also be required to manage adverse events (AEs). The use fuzuloparib is contraindicated during breastfeeding; do not breastfeed during treatment and for one month after the last dose of fuzuloparib [3]. 


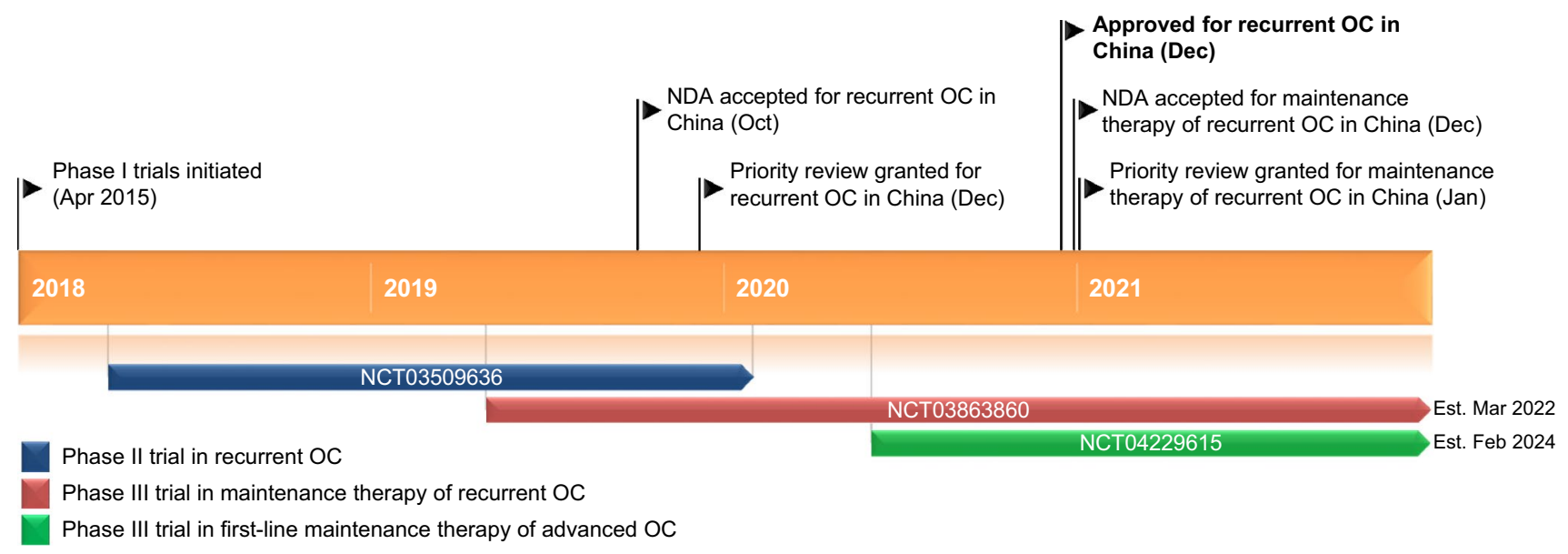

Key milestones in the development of fuzuloparib for the treatment of OC. NDA New drug application, $O C$ ovarian cancer

\section{Scientific Summary}

\subsection{Pharmacodynamics}

Inhibition of the PARP1 enzyme with fuzuloparib was comparable with olaparib (PARP1 IC I0 $1.46 \mathrm{nM}$ with fuzuloparib and $1.34 \mathrm{nM}$ with olaparib) when measured using ELISA [4]. Both fuzuloparib and olaparib inhibited $B R C A 1$ or $B R C A 2$-deficient lung fibroblast, ovarian cancer and breast cancer cell lines in cell culture experiments (IC $_{50} 0.053-1.57 \mu \mathrm{M}$ with fuzuloparib and $0.035-2.16 \mu \mathrm{M}$ with olaparib); though no relevant inhibition of $B R C A 1$ or $B R C A 2$-positive cell lines were observed with both inhibitors ( $\mathrm{IC}_{50}>10 \mu \mathrm{M}$ with both inhibitors). In animal studies, fuzuloparib $30 \mathrm{mg} / \mathrm{kg}$ and olaparib $30 \mathrm{mg} / \mathrm{kg}$ inhibited the growth of BRCAl-negative breast cancer in mice (day 21 inhibition rate $59 \%$ with fuzuloparib and $44 \%$ with olaparib). Neither PARP inhibitor was associated with significant differences in body weight compared with placebo treated mice [4].

PARP inhibition is known to induce embryo-foetal toxicity in animal studies, and fuzuloparib may cause foetal harm in pregnant women. Contraception is recommended in women of childbearing age during treatment and for 6 months after the last dose of fuzuloparib [3].

\subsection{Pharmacokinetics}

A dose-proportional increase in the $\mathrm{AUC}_{0-\mathrm{t}}$ and $\mathrm{C}_{\max }$ of fuzuloparib occurs across a dose range of $10-150 \mathrm{mg}$ when administered as a single dose. The accumulation ratio (in $\mathrm{AUC}_{0-t}$ ) after 13 days is 1.86 in patients receiving fuzuloparib $150 \mathrm{mg}$ twice daily. The administration of fuzuloparib after a high-fat meal does not have a significant effect on the AUC, though the $t_{\max }$ is delayed from $3 \mathrm{~h}$ to $6 \mathrm{~h}$. The apparent volume of distribution of fuzuloparib is $34.6 \mathrm{~L}$.
Plasma binding of fuzuloparib is $74.3-81.6 \%$ over a concentration range of $20-2000 \mathrm{ng} / \mathrm{mL}$. The terminal half-life of fuzuloparib is $9.14 \mathrm{~h}$ in patients administered multiple doses of fuzuloparib $150 \mathrm{mg}$ twice daily. Fuzuloparib is predominantly metabolised by CYP3A4, with the most common metabolites being mono-oxidation and subsequently hydrogenated products; each metabolite contributes $<10 \%$ of the total plasma radioactivity. $44.2 \%$ and $59.1 \%$ of the radioactivity from the original dose is excreted into the faeces and urine. $15.8-16.8 \%$ of the original dose is excreted as unchanged fuzuloparib in urine [3].

Concomitant administration of itraconazole (strong CYP3A4 inhibitor) increases the $\mathrm{C}_{\max }, \mathrm{AUC}_{0-\mathrm{t}}$ and $\mathrm{AUC}$ ${ }_{\infty}$ of fuzuloparib by $51.0 \%, 325 \%$ and $381 \%$, respectively; increases of $32.4 \%, 104.5 \%$ and $109.6 \%$, respectively, are observed with fluconazole (moderate CYP3A4 inhibitor). Induction of CYP3A4 by rifampicin decreases the $\mathrm{C}_{\max }$, $\mathrm{AUC}_{0-\mathrm{t}}$ and $\mathrm{AUC}_{\infty}$ of fuzuloparib to $32.0 \%, 10.4 \%$, and $10.4 \%$, respectively. Inhibition of the CYP1A2, CYP2B6, CYP2C8, CYP2C9, CYP2C19, CYP2D6 and CYP3A4 enzymes by fuzuloparib poses a low risk for drug-drug interactions, and no drug-drug inhibition studies are required according to relevant guidelines. Fuzuloparib may induce CYP1A2, CYP2B6 and CYP3A4 at a concentration of $5 \mu \mathrm{M}$, based on data from in vitro studies. Clinical<smiles>O=C(c1cc(Cc2n[nH]c(=O)c3ccccc23)ccc1F)N1CCn2nc(C(F)(F)F)nc2C1</smiles>

Chemical structure of fuzuloparib 
studies to investigate the induction of CYP1A2, CYP2B6, CYP2C8, CYP2C9, CYP2C19 and CYP3A4 by fuzuloparib are ongoing. Fuzuloparib is not recommended in patients with moderate or severe hepatic impairment or renal impairment as data are not yet available in these patient populations. Mild hepatic impairment and mild renal impairment does not cause relevant changes in clearance of fuzuloparib [3].

\subsection{Therapeutic Trials}

\subsubsection{Ovarian Cancer}

The first approval of fuzuloparib in the treatment of recurrent ovarian cancer was granted following the results from a phase II, open-label trial (NCT03509636). This trial enrolled patients with platinum-sensitive, recurrent, BRCA1/2-mutant, high-grade serous or endometrioid ovarian cancer who had previously received 2-4 lines of platinum-based chemotherapy. 113 patients were treated with fuzuloparib $150 \mathrm{mg}$ twice daily in 28-day cycles. Fuzuloparib achieved an independent review committee (IRC)-assessed objective response rate (ORR) of $69.9 \%$ (primary endpoint); with $4.4 \%$ of patients achieving a complete response (CR) and $65.5 \%$ with a partial response (PR). The median follow-up duration was 15.9 months as of the latest data cut-off (21 Mar 2020) [5].

Fuzuloparib was investigated as a maintenance therapy for recurrent ovarian cancer in a randomised phase III trial
(NCT03863860) in patients with platinum-sensitive, recurrent, high-grade serous or endometrioid ovarian cancer who received at least two previous lines of platinum-based chemotherapy and achieved either CR or PR to their most recent regimen. 167 patients were randomised to receive fuzuloparib $150 \mathrm{mg}$ twice daily in 28-day cycles, and 85 patients were assigned to receive placebo. Fuzuloparib significantly $(p<0.0001)$ prolonged blinded independent review committee (BIRC)-assessed progression-free survival (PFS) versus placebo in the overall trial population. The median BIRC-assessed PFS was 12.9 and 5.5 months in the fuzuloparib and placebo treatment groups [hazard ratio (HR) 0.25 ; $95 \%$ confidence interval (CI) 0.17-0.36], though the median PFS for fuzuloparib was an estimate as data are not yet mature. Furthermore, fuzuloparib was superior to placebo in extending BIRC-assessed PFS in a subgroup of patients with $g B R C A m$ [HR 0.14; 95\% CI 0.07-0.28] (co-primary endpoints). As of the latest data cut-off date (1 Jul 2020), $67 \%$ of planned PFS events occurred in the overall population and the median follow-up duration was 8.5 months [6].

\subsubsection{Other Solid Cancers}

Fuzuloparib as a monotherapy in advanced solid tumours was examined in a phase I study (NCT02575651). 79 patients who had advanced solid tumours (including ovarian, breast and colorectal cancers) which were refractory to standard treatment or cancers which did not have a standard treatment were enrolled in this study. Patients were treated with fuzuloparib

\section{Features and properties of fuzuloparib}

\begin{tabular}{|c|c|}
\hline Alternative names & Fluzoparib, AiRuiYi ${ }^{\circledR}$, 艾瑞虍, 氟唑帕利胶囊, HS10160, SHR3162 \\
\hline Class & $\begin{array}{l}\text { 2-ring heterocyclic compounds, antineoplastics, fluorobenzenes, phthalazines, pyrazines, small molecules, } \\
\text { triazoles }\end{array}$ \\
\hline Mechanism of action & $\begin{array}{l}\text { Small molecule PARP inhibitor; inhibition of DNA repair pathways leads to cell cycle arrest and prevents the } \\
\text { proliferation of tumour cells }\end{array}$ \\
\hline Route of administration & Oral \\
\hline Pharmacodynamics & 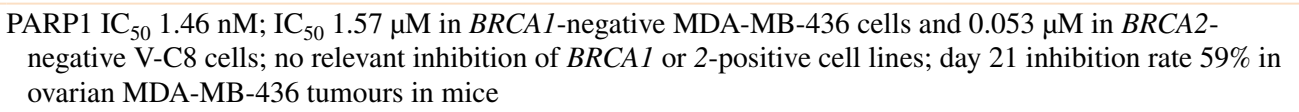 \\
\hline Pharmacokinetics & $\begin{array}{l}\text { Proportional increases in } \mathrm{AUC}_{0-\mathrm{t}} \text { and } \mathrm{C}_{\mathrm{max}} \text { with single fuzuloparib doses between } 10-150 \mathrm{mg} \text {; accumulation } \\
\text { ratio of } 1.86 \text { after } 13 \text { days of receiving fuzuloparib } 150 \mathrm{mg} \text { twice daily; } \mathrm{V}_{\mathrm{d}} 34.6 \mathrm{~L} ; 74.3 \%-81.6 \% \text { human } \\
\text { plasma protein binding; mainly metabolised by CYP3A4; most common metabolites were mono-oxidation } \\
\text { and subsequently hydrogenated products }\left(<10 \% \text { each in plasma); } \mathrm{t}_{1 / 2} 9.14 \mathrm{~h} ; \sim 60 \% \text { of the dose is excreted in }\right. \\
\text { urine and } \sim 40 \% \text { in faeces; } \sim 16 \% \text { of the dose is excreted in urine as unchanged drug }\end{array}$ \\
\hline \multicolumn{2}{|l|}{ Most frequent adverse events } \\
\hline $\begin{array}{l}\text { All-grade events } \\
\quad \text { (incidence } \geq 40 \%)\end{array}$ & Anaemia, nausea, leukopenia, fatigue and thrombocytopenia \\
\hline $\begin{array}{l}\text { Most frequent grade } \geq 3 \text { events } \\
\quad \text { (incidence } \geq 2 \% \text { ) }\end{array}$ & Anaemia, thrombocytopenia, neutropenia, leukopenia and lymphopenia \\
\hline \multicolumn{2}{|l|}{ ATC codes } \\
\hline WHO ATC code & L01X-X \\
\hline EphMRA ATC code & L1X9 \\
\hline Chemical name & $\begin{array}{l}\text { 4-[[4-fluoro-3-[2-(trifluoromethyl)-6,8-dihydro-5H-[1,2,4]triazolo[1,5-a]pyrazine-7-carbonyl]phenyl] } \\
\text { methyl]-2H-phthalazin-1-one }\end{array}$ \\
\hline
\end{tabular}


dosages ranging from $10 \mathrm{mg}$ once daily to $200 \mathrm{mg}$ twice daily. An ORR of 6.2\% (all PR) and a disease control rate of $30.8 \%$ at 24 weeks was reported in 65 evaluable patients [7].

In a dose-escalation phase I study (NCT03026881), 39 patients with advanced gastric or gastroesophageal junction cancer who did not respond to platinum-based chemotherapy were treated with a combination of fuzuloparib, apatinib and paclitaxel. 12 of 36 evaluable patients achieved a confirmed PR and 13 patients had stable disease following combination treatment [8].

\subsection{Adverse Events}

Safety data on monotherapy with fuzuloparib $150 \mathrm{mg}$ twice daily were collected from three studies that enrolled a total of 294 patients with ovarian cancer [3]. All-grade AEs were reported in $94.9 \%$ of patients, with the most common AEs with an incidence $\geq 40 \%$ being anaemia (58.5\% of patients), nausea (57.1\%), leukopenia (54.4\%), fatigue (43.5\%) and thrombocytopenia (41.5\%). The most common grade $\geq 3$ AEs with an incidence $\geq 2 \%$ were haematological AEs; anaemia (25.5\%), thrombocytopenia (13.3\%), neutropenia (10.5\%), leukopenia (9.9\%) and lymphocytopenia (5.1\%). The overall incidence of grade $\geq 3$ haematological AEs was $40.5 \%$. Dose interruption or reduction were required in some patients due to anaemia (dose interruption in $23.1 \%$ of patients and dose reduction in $9.2 \%$ of patients), thrombocytopenia (13.9\% and 6.5\%), neutropenia (8.8\% and 2.0\%), leukopenia (9.5\% and 3.4\%) and lymphocytopenia ( $2.0 \%$ and $0.7 \%$ ). Monitor complete blood count at baseline then every fortnight for three months after initiating treatment and regularly afterwards. Consult local prescribing information for recommendations on dose reduction or interruption. Cases of myelodysplastic syndrome (MDS) or acute myeloid leukaemia (AML) have been reported during clinical trials in other PARP inhibitors used for the treatment of cancer. If signs and symptoms of MDS or AML are observed, discontinue fuzuloparib treatment and administer appropriate therapy [3].

Gastrointestinal AEs have been reported with fuzuloparib, with an overall all-grade incidence of $72.8 \%$ (2.4\% for grade $\geq 3)$. The incidence of all-grade nausea was $57.1 \%(0.3 \%$ for grade $\geq 3$ ) and $26.2 \%$ (1.4\% for grade $\geq 3$ ) for vomiting. Grade $\geq 2$ nausea and vomiting may be managed with dose interruption, dose reduction and/or antiemetic medications [3].

\subsection{Ongoing Clinical Trials}

Fuzuloparib is being investigated in phase II and III trials in various solid cancers. There are four trials of fuzuloparib in ovarian cancer, NCT03863860 for maintenance therapy in patients with recurrent platinum-sensitive ovarian cancer who received at least two previous lines of platinum-based chemotherapy and achieved either CR or PR to their most recent regimen (phase III; fuzuloparib vs placebo); NCT04229615 for maintenance treatment in patients with advanced ovarian cancer following response on first-line platinum-based chemotherapy (phase III; fuzuloparib with/without apatinib vs placebo); NCT04517357 in patients with high-grade serous or endometrioid ovarian cancer who completed two or more platinum-containing regimens (phase II; fuzuloparib monotherapy vs fuzuloparib plus apatinib) and NCT03509636 in patients with platinum-sensitive BRCA1/2-mutant disease who received 2-4 chemotherapy regimens (phase II; fuzuloparib arm only).

Two trials are assessing fuzuloparib in pancreatic cancer, NCT04300114 for maintenance therapy in patients with $g B R C A / P A L B 2$-mutatant metastatic pancreatic cancer whose disease has not progressed following first-line platinum-based chemotherapy (phase III; fuzuloparib vs placebo) and NCT04228601 for combination therapy and subsequent maintenance therapy in advanced pancreatic cancer (phase I/II; fuzuloparib plus mFOLFIRINOX followed by fuzuloparib maintenance therapy vs placebo plus mFOLFIRINOX followed by placebo maintenance therapy).

Three trials are evaluating fuzuloparib in metastatic castration-resistant prostate cancer, NCT04691804 for combination therapy as a first-line treatment (phase III; fuzuloparib plus abiraterone acetate and prednisone vs placebo plus abiraterone acetate and prednisone), NCT04869488 for combination therapy or monotherapy in patients who failed prior treatment with abiraterone/enzalutamine and with or without homologous recombination repair gene mutations (phase II; fuzuloparib with/without apatinib vs abiraterone/ enzalutamine plus prednisone) and NCT04102124 for combination therapy in patients who did not respond to abiraterone and docetaxel treatment (phase II; fuzuloparib plus rezvilutamide vs rezvilutamide plus placebo vs placebo).

Other trials of fuzuloparib includes NCT04296370 in patients with $H E R 2$-negative metastatic breast cancer with $g B R C A m$ who received no more than two lines of chemotherapy, received prior therapy with an anthracycline and a taxane and did not respond to one endocrine therapy or are not candidates for endocrine therapy (phase III; fuzuloparib with/without apatinib vs chemotherapy) and NCT04400188 combination therapy in patients with relapsed small cell lung cancer who failed at least one line of platinum-based chemotherapy (phase I/II; fuzuloparib plus temozolomide with/without adebrelimab).

\section{Current Status}

Fuzuloparib received its first approval on 11 Dec 2020 for treatment of platinum-sensitive recurrent ovarian cancer, fallopian tube cancer or primary peritoneal cancer in patients with $g B R C A m$ who have undergone second-line or above chemotherapy in China [1-3]. 


\begin{tabular}{|c|c|c|c|c|}
\hline $\operatorname{Drug}(\mathrm{s})$ & Indication & Phase & Status & Identifier \\
\hline \multicolumn{5}{|l|}{ Ovarian cancers } \\
\hline Fuzuloparib, placebo & $\begin{array}{l}\text { Maintenance therapy in platinum-sensitive recurrent ovar- } \\
\text { ian cancer after at least two previous lines of platinum-based } \\
\text { chemotherapy and achieved either CR or PR to their most recent } \\
\text { regimen }\end{array}$ & III & Active & NCT03863860 \\
\hline $\begin{array}{l}\text { Fuzuloparib, apatinib, } \\
\text { placebo }\end{array}$ & $\begin{array}{l}\text { Maintenance therapy in advanced ovarian cancer following } \\
\text { response on first-line platinum-based chemotherapy }\end{array}$ & III & Recruiting & NCT04229615 \\
\hline Fuzuloparib, apatinib & $\begin{array}{l}\text { Advanced ovarian cancer following } 2 \text { or more platinum-contain- } \\
\text { ing regimens }\end{array}$ & II & Recruiting & NCT04517357 \\
\hline Fuzuloparib & $\begin{array}{l}\text { Platinum-sensitive, } B R C A 1 / 2 \text {-mutant recurrent ovarian cancer } \\
\text { following } 2 \text { or more chemotherapy regimens }\end{array}$ & II & Active & NCT03509636 \\
\hline \multicolumn{5}{|l|}{ Pancreatic cancers } \\
\hline Fuzuloparib, placebo & $\begin{array}{l}\text { Maintenance therapy in patients with } g B R C A / P A L B 2 \text {-mutant } \\
\text { metastatic pancreatic cancer whose disease has not progressed } \\
\text { following first-line platinum-based chemotherapy }\end{array}$ & III & Recruiting & NCT04300114 \\
\hline $\begin{array}{l}\text { Fuzuloparib, placebo, } \\
\text { mFOLFIRINOX }\end{array}$ & $\begin{array}{l}\text { Combination therapy and subsequent maintenance therapy in } \\
\text { advanced pancreatic cancer }\end{array}$ & $\mathrm{I} / \mathrm{II}$ & Recruiting & NCT04228601 \\
\hline \multicolumn{5}{|l|}{ Prostate cancers } \\
\hline $\begin{array}{l}\text { Fuzuloparib, placebo, } \\
\text { abiraterone acetate and } \\
\text { prednisone }\end{array}$ & $\begin{array}{l}\text { First-line treatment in metastatic castration-resistant prostate } \\
\text { cancer }\end{array}$ & III & Not yet recruiting & NCT04691804 \\
\hline $\begin{array}{l}\text { Fuzuloparib, abiraterone, } \\
\text { apatinib, enzalutamine, } \\
\text { prednisone }\end{array}$ & $\begin{array}{l}\text { Metastatic castration-resistant prostate cancer in patients who } \\
\text { failed prior treatment with abiraterone/enzalutamine and with } \\
\text { or without homologous recombination repair gene mutations }\end{array}$ & II & Not yet recruiting & NCT04869488 \\
\hline $\begin{array}{l}\text { Fuzuloparib, rezviluta- } \\
\text { mide, placebo }\end{array}$ & $\begin{array}{l}\text { Metastatic castration-resistant prostate cancer patients who did } \\
\text { not respond to abiraterone and docetaxel treatment }\end{array}$ & II & Active & NCT04102124 \\
\hline \multicolumn{5}{|l|}{ Breast cancers } \\
\hline $\begin{array}{l}\text { Fuzuloparib, apatinib, } \\
\text { chemotherapy }\end{array}$ & $\begin{array}{l}\text { HER2-negative metastatic breast cancer with germline } B R C A \\
\text { mutation in patients who received no more than two lines of } \\
\text { chemotherapy, received prior therapy with an anthracycline and } \\
\text { a taxane and did not respond to one endocrine therapy or are } \\
\text { not candidates for endocrine therapy }\end{array}$ & III & Recruiting & NCT04296370 \\
\hline \multicolumn{5}{|l|}{ Lung cancers } \\
\hline $\begin{array}{l}\text { Fuzuloparib, adebrelimab, } \\
\text { temozolomide }\end{array}$ & $\begin{array}{l}\text { Relapsed small cell lung cancer in patients who failed one line of } \\
\text { platinum-based chemotherapy }\end{array}$ & $\mathrm{I} / \mathrm{II}$ & Recruiting & NCT04400188 \\
\hline
\end{tabular}

Supplementary Information The online version contains supplementary material available at https://doi.org/10.1007/s40265-021-01541-x.

\section{Declarations}

Funding The preparation of this review was not supported by any external funding.

Authorship and Conflict of interest During the peer review process the manufacturer of the agent under review was offered an opportunity to comment on the article. Changes resulting from any comments received were made by the authors on the basis of scientific completeness and accuracy. Arnold Lee is a salaried employee of Adis International Ltd/ Springer Nature, and declares no relevant conflicts of interest. All authors contributed to the review and are responsible for the article content.
Ethics approval, Consent to participate, Consent to publish, Availability of data and material, Code availability Not applicable.

Open Access This article is licensed under a Creative Commons Attribution-NonCommercial 4.0 International License, which permits any non-commercial use, sharing, adaptation, distribution and reproduction in any medium or format, as long as you give appropriate credit to the original author(s) and the source, provide a link to the Creative Commons licence, and indicate if changes were made. The images or other third party material in this article are included in the article's Creative Commons licence, unless indicated otherwise in a credit line to the material. If material is not included in the article's Creative Commons licence and your intended use is not permitted by statutory regulation or exceeds the permitted use, you will need to obtain permission directly from the copyright holder. To view a copy of this licence, visit http://creativecommons.org/licenses/by-nc/4.0/. 


\section{References}

1. Jiangsu Hengrui Pharmaceuticals Co., Ltd. China's first original PARP inhibitor fluzoparib approved for marketing [media release]. 17 Dec 2020. https://www.hrs.com.cn.

2. National Medical Products Administration. Drug Evaluation Center. The National Medical Products Administration approves the marketing of fluzoparib capsules [media release]. $14 \mathrm{Dec}$ 2020. https://www.nmpa.gov.cn/directory/web/nmpa/yaopin/ ypjgdt/20201214100312149.html.

3. Jiangsu Hengrui Pharmaceuticals Co., Ltd. AiRuiYi ${ }^{\circledR}$ (fluzoparib) capsules: Chinese prescribing information [Chinese]. Lianyungang: Jiangsu Hengrui Pharmaceuticals Co., Ltd.; 2020.

4. Wang L, Yang C, Xie C, et al. Pharmacologic characterization of fluzoparib, a novel poly(ADP-ribose) polymerase inhibitor undergoing clinical trials. Cancer Sci. 2019;110(3):1064-75.
5. Li N, Bu H, Liu J, et al. An open-label, multi-center, single-arm, phase 2 study of fluzoparib in patients with germline BRCA1/2 mutation and platinum-sensitive recurrent ovarian cancer. Clin Cancer Res. 2021. https://doi.org/10.1158/1078-0432. CCR-20-3546.

6. Li N, Zhang Y, Zhu J. Fuzuloparib maintenance therapy in patients with platinum-sensitive, recurrent ovarian carcinoma: a multicenter, randomized, double-blind, placebo-controlled, phase III trial [abstract no. 11557 plus oral presentation]. In: SGO 2021.

7. Li H, Liu R, Shao B, et al. Phase I dose-escalation and expansion study of PARP inhibitor, fluzoparib (SHR3162), in patients with advanced solid tumors. Chin J Cancer Res. 2020;32(3):370-82.

8. Xu JM, Liu R, Ba Y, et al. Phase I study of fluzoparib, a PARP1 Inhibitor in combination with apatinib and paclitaxel in patients (pts) with advanced gastric and gastroesophageal junction (GEJ) adenocarcinoma [abstract no. 4060]. J Clin Oncol. 2019;37(Suppl. 15):4060. 